\title{
Erratum to: Modeling organic carbon and carbon-mediated soil processes in DSSAT v4.5
}

\author{
Cheryl H. Porter · J. W. Jones • S. Adiku •
}

A. J. Gijsman • O. Gargiulo • J. B. Naab

Published online: 8 March 2014

(C) Springer-Verlag Berlin Heidelberg 2014

\section{Erratum to: Oper Res Int J (2010) 10:247-278 DOI 10.1007/s12351-009-0059-1}

When we published the above-mentioned article, the equation 7 was not given correctly. Instead of

$$
\theta_{\mathrm{SATm}}=S_{m} \cdot M_{\mathrm{mulch}} \cdot 10^{-4}
$$

it should read

$$
\Delta \text { Dlayr }=\frac{\Delta S O M}{B D_{S O M}} * \frac{10^{-5} \mathrm{~g} / \mathrm{cm}^{2}}{\mathrm{~kg} / \mathrm{ha}}
$$

The online version of the original article can be found under doi:10.1007/s12351-009-0059-1.

C. H. Porter $(\bowtie) \cdot$ J. W. Jones · A. J. Gijsman · O. Gargiulo

Department of Agricultural and Biological Engineering,

University of Florida, 103 Frazier Rogers Hall, PO Box 110570,

Gainesville, FL 32611-0570, USA

e-mail: cporter@ufl.edu

S. Adiku

Agricultural Systems Research Unit, USDA, Ft. Collins, CO, USA

J. B. Naab

Savannah Agricultural Research Institute, Wa, Ghana 